\title{
Automatic Temperature Measurement for Hot Spots in Face Region of Cattle using Infrared Thermography
}

\author{
Mohammed Ahmed Jaddoa ${ }^{1}$, Adel Ahmed Al-Jumaily ${ }^{1}$, \\ Luciano Adrian Gonzalez ${ }^{2}$ and Holly Cuthbertson ${ }^{2}$ \\ ${ }^{I}$ Faculty of Engineering and IT, University of Sydney Technology (UTS), 15 Broadway, Ultimo NSW, Australia \\ ${ }^{2}$ Faculty of Agriculture and Environment, University of Sydney, Ultimo NSW, Australia \\ Mohammed.A.Jaddoa@student.uts.edu.au,adel.al-jumaily@uts.edu.au, \\ luciano.gonzalez@sydney.edu.au, holly.cuthbertson@sydney.edu.au
}

\begin{abstract}
Keywords: Infrared Thermal, Automatic Hot Spot Extraction, Face Detection in Cattle, Temperature Measurement in Cattle.

Abstract: Infrared Thermography Technology (IRT) is a non-invasive method that has been used to calculate and display temperature as an Infrared thermal image. Infrared thermal images are used frequently to measure temperature remotely, and this temperature can be used as a health indicator for detecting diseases and inflammation in human and animal. In cattle, the rising temperature of the eye and nose region used for identifying stress and Bovine respiratory disease (BRD). In such applications, measuring temperature for nose and eye region is conducted manually. In this paper, a new automatic method is proposed for extracting the hottest regions from the face region, which may include eyes, nose and mouth. The proposed method involves face detection, thresholding, and blob refinement. The preliminary results show that the proposed algorithm is working well for localization and temperature measurement.
\end{abstract}

\section{INTRODUCTION}

Infrared Thermography Technology (IRT) is a noninvasive method that has been used to calculate and display temperature as an image. IRT can detect the variations in temperature and detect blood flow through determining the changes in body temperature (Nääs, Garcia et al. 2014, Roberto, de Souza et al. 2014). The concept behind IRT is measuring heat radiation that emitted from the surface of objects (Roberto, de Souza et al. 2014). There are some restrictions associated with this technology that needs from users to care about while taking an image by using IRT camera. These restrictions include sunlight, high humidity as well as heat loss because of the wind or when the surface of the body is unclean. Also, the emissivity of radiation for objects and reflection (connectivity with another object) of radiation that comes from other object are also considered as other factors that affected the accuracy of captured results by an infrared camera (Nääs, Garcia et al. 2014). Other significant factors or parameters that required providing to the camera were the distance between the object and camera and humidity (Rekant, Lyons et al. 2016). Even though the obstacles of using IRT, IRT has been applied widely in different area such as veterinary medicine.

The common usage of IRT is its ability in providing temperature remotely without need from human to be close from target animal. Extracted temperature can be used for monitoring and evaluating animal health for early detection of rising body temperature which is a sign of fever or local inflammation. As examples for IRT application in veterinary medicine, IRT has succeeded in detection different disease such as mastitis in dairy cows (Polat, Colak et al. 2010). The rising temperature of udder used as an indicator of mastitis detection. Another utilization of IRT is the ability for discovering the increase of temperature which is used for inflammation identification in cows (Pezeshki, Stordeur et al. 2011). Also, IRT used for stress detection in cows through temperature analysis for eyes region (Stewart, Webster et al. 2007) and used the same region as an indicator for feed efficiency in cows (Palme and Schenkel). Bovine respiratory disease (BRD) in calves can be detected in early stage through IRT by measuring temperature for eyes region (Schaefer, Cook et al. 2007). One of the 
significant signs of BRD disease is increasing temperature for eyes region during infection. Inflammation, stress and diseases succeed effectively in Equine Medicine by temperature analysis of infrared thermal images (Soroko and Howell 2018). In pigs, a rising surface temperature of the lacrimal caruncle (ocular), auricular pavilion and nose area of weaned piglets were used to find impacts the effects of environmental enrichment and social structure (Yáñez-Pizaña, Mota-Rojas et al. 2019). However, extracting temperature by using infrared thermography was studied extensively as a result of it is significant. The utilization of IRT in veterinary medicine is crucial and useful in detecting cattle disease and inflammation that is able to treat it in the early stage before it can spread to other animals in a herd.

Previous studies were achieved through including trained personnel to use a computer connected to infrared thermal camera (Rekant, Lyons et al. 2016). This means to identify a particular spot manually in face region and other organ of the animal body. In this paper, a new method is proposed for extracting hottest spots from animal face through using machine learning and image processing. Based on machine learning, face region was identified automatically. While in image processing, thresholding, and blob refinement used for extracting hot spot of a face region and measure temperature for extracted spots. Based on high temperature, these spots may include the nose, eyes and mouth.

The structure of the paper is as the following: Related work presented in Sect. 2. The database is described in Sect. 3, followed by the proposed algorithm with its subsections in Sect. 4. The paper is concluded by a result discussion (Sect. 5) and a conclusion (Sect. 6).

\section{RELATED WORK}

Some selected methods of temperature extraction and analysis for face region from thermal images are presented and discussed below.

The authors in publication ( $\mathrm{Li}$, Menassa et al. 2018) used infrared thermal images in order to measure the thermal comfort of people to modify operational setting Heating, Ventilation and Air Conditioning (HVAC) systems in buildings. Skin temperature measured from facial regions after applying face detection in frontal face position by applying Haar Cascade algorithm. In the mentioned study, the proposed method was validated through including twelve of subjects for testing infrared comfort. The results have shown that ear, nose and cheek are best indicators to assets infrared thermal comfort, and it is accurate with the percentage of $85 \%$. Haar Cascade algorithm required a lot of images and need to adjust the size of the bounding box as well as false detection, which make it an undesirable choice.

In this research paper (Jaddoa, Al-Jumaily et al. 2017) used infrared thermal images for eye localization in cattle based on ellipse detection and image processing. Randomized Hough Transform algorithm was used for ellipse detection. Ellipse detection was used in this study to localize eye region, and thresholding used to extract eyes. Results showed that proposed method has good performance in eye localization in different orientation and localization. This method is not appropriate when the direction of animal face lead to a change in an ellipse shape. In addition, it is hard to apply to detect eye region as a result of overlapping of different animal faces at the same time.

(Cruz-Albarran, Benitez-Rangel et al. 2017), reported that the infrared thermal was used as an image source for developing a smart thermal system to diagnose emotions in human. After using the reading for the ambient temperature, and emissivity value, the static temperature was used as a threshold value in order to extract face region. Emotion identification achieved through temperature analysis for the nose, forehead, nose and maxillary from detected face region. In this study, 44 subjects participated and the method has the ability to detection emotion with $89.9 \%$ percentage.

While in other work (Budzan and Wyżgolik 2013), eyes and face detection as well as canthus region of detected eye implemented on infrared thermal images for temperature measurement purpose. For face detection, Randomized Hough transform was used to identify face region. The combination of template-matching, knowledge-based and morphological methods employed for eye and eye-corner detection. The researchers of this paper pointed out that the proposed method accurate with percentage 97 with distance range 1.50 to $3.0 \mathrm{~m}$ between camera and subject.

As reported above, the methods described in the literature referred hardly to the issue of temperature analysis for face region in cattle. Secondly, all methods start by identifying face region before going to temperature analysis. Temperature analysis is crucial for diseases and inflammation diagnosis as mention in the introduction section. High intensity in the infrared thermal image means rising temperature, and high intensity is an indicator of inflammation and 
infection. Thus, there is no research done regarding the automated extracting temperature of the hot spot of the animal face. To overcome the research gaps identified in the existing body of knowledge, it is critical to have a non-intrusive method for temperature analysis of hot spots of face region in cattle.

The aim of this paper is to propose a new method for temperature analysis of hot spots from face region in the frontal position. The proposed method includes face detection using linear support vector machine (SVM) and Histograms of Oriented Gradients (HOG) features and a new image processing method for hot spot extraction, and temperature measurement as final results.

\section{DATA COLLECION AND CLEANING}

The Infrared Thermography database used for testing the proposed system was created through converting video with seq format to a sequence of grey images and infrared matrix. Infrared matrix created based on equation (1).

$$
T_{c}=T_{\text {min }}+\left(\frac{T_{\text {gray }}}{T_{\text {High_gray }}}\left(T_{\text {max }}-T_{\text {min }}\right)\right)
$$

Where $T_{c}$ is calculated temperature according to thermal value of the infrared thermal image. $T_{\min }$ and $T_{\max }$ refer to maximum and the minimum temperature value of the thermal camera. $T_{\text {gray }}$ represent pixel intensity of grey image. $T_{\text {High_gray }}$ is the high intensity of grey image which it in most cases is 255 . The utilization of this calculation leads to generate an infrared matrix with a temperature range of 20 to 38 Celsius.

The infrared thermal images collection included 150 thermograms image show animal face in frontal position. The captured infrared thermal video recorded in Arthursleigh Farm that it belongs to Sydney University. It is located in Marulan town in the Southern Tablelands of New South Wales, Australia.

Duration of the video is two hours involving 73 animals in different positions and orientation. There is no any kind of manipulation in the background, which means this data is represent the real scenario of animals roaming in the farm. Animal face in the frontal position used in this study. The images were acquired by using AGEMA 590 PAL, ThermaCam S65, A310, T335 with $320 \times 240$-pixel for resolution. Infrared image and matrix have same resolution.The distance between camera and subject was about 1.50 to $3 \mathrm{~m}$. Fig.3 (A,B,C,D) shows a sample of the prepared infrared thermal database, which includes a herd of cattle indoor environment in the farm.

\section{METHODOLOGY}

The proposed method for temperature extraction and analysis for face region has the following components as illustrated in a Fig.1. As seen in Fig.1, the proposed method starting by loading an infrared image in a grey format and infrared thermal matrix. In the grey image, removing noise and histogram equalization applied as a pre-processing process before face detection stage. Face detection applied to identify Region of Interest (ROI) from an input image. Cropping of the face region and binarization implemented to identify important spot from detected face. In Blob refinement stage, temperature from each blob extracted to keep only hot spots from face. The last process stage is temperature measurement. Each process stage of the proposed method will be explained in details in the next sections.

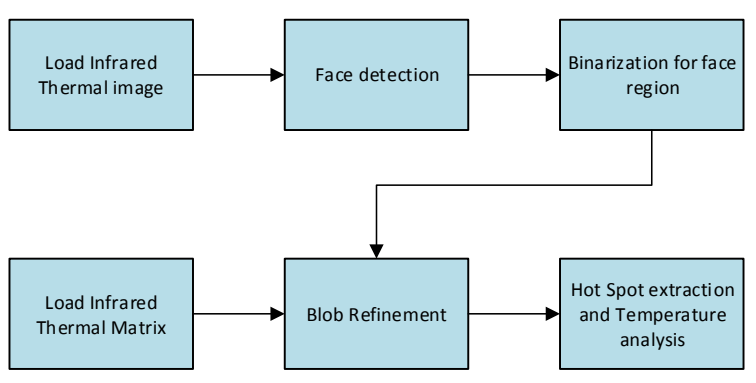

Figure 1: Proposed method components.

\subsection{Face Detection}

Due to the important role of infrared thermal imaging in monitoring and medical sector, several methods for face detection in thermal infrared images have been developed. These methods use state-of-the-art processes from machine learning and feature extraction to detect faces in images and videos. A recent study compares the performance of different face detection algorithm using infrared thermal images of human (Kopaczka, Nestler et al. 2017). Face detection algorithms include Haar cascade classifier (VJ), Haar cascade classifier with local binary patterns (VJ-LBP), Histograms of Oriented Gradients (HOG) and SVM, The Deformable Parts Model (DPM), Pixel Intensity Comparisons Organized in Decision Trees (PICO), Eye Corner Detection (ED) and Projection Profile Analysis 
(PPA)(Kopaczka, Nestler et al. 2017). Based on the experimental results, the performances of theses algorithm in term of precision are $\mathrm{VJ}(0.93), \mathrm{VJ}-$ LBP(0.97),HOG(1.00),PICO(0.90),PPA(0.85),ED(0. 52) and DPM(1.00).

The main concept behind these algorithms is an automated classification for extracted features from the image. Compared to the visual spectrum, there is a lack of literature using face detection approach specially developed for the thermal infrared spectrum in animals. The reason for this lack is automated classification based on machine learning requires extensive amounts of manually annotated training data which is not available for the thermal infrared domain. In this paper, the researchers' own data were used for training and testing. In addition, HOG for feature extraction and SVM for classification will be used in this paper for face detection.

Histograms of Oriented Gradients (HOG) is an image features representation for image based on the computational calculation of intensity gradient of an image which presented by Dalal and Triggs (Dalal and Triggs 2005). Currently, it is one of the most widely used methods for object detection. In our work for face detection, HOG is computed and converted as a feature vector, and the feature vector is computed for a training set of face and non-face images and the results are used to train a support vector machine (SVM). SVM learns how to distinguish the HOG feature representation of a face from background features.

In our work, we used the implementation available in the dlib library (King 2009) to train and test a HOG-based face detector. 150 infrared thermal images used for training and 150 images also used for testing. In training stage, face region of cattle was labelled manually through using labeller app in matlab. This small amount of images was used for training because it required spending a lot of time in manual labelling. In testing stage, another 150 of image used without labelling to test the ability of trained face detector.

After applying face detection, a binary converting for face region will be used for extracting hot spot from face region as shown in the next section.

\subsection{Binarization for Face Region}

After applying, face detection, cropping face region based on the coordination of bounding box. After applying, face detection, cropping face region based on the coordination of bounding box. Histogram equalization applied on pixels intensity of cropped image. The aim of histogram equalization step is to normalize image brightness and contrast through modifying pixels intensity by using histogram distribution. The concept behind histogram equalization is scaling the most frequent pixel intensity value compare to other pixels values. Fig.2 present examples of image before and after apply histogram equalization. As seen in fig.2, eyes and nose of animal face become brighter with pixel intensity reach to 255 . In contrast, dark region become darker with pixels intensity close from 0 .

After applying Histogram equalization, thresholding used for converting cropped face region to a binary image. 250 used as thresholding value because all regions with high and medium brightness become brighter that mean it is close from 255(fig.2(C)). Threshold value identifies based on the equation (2).

$$
B(x, y)=\left\{\begin{array}{rr}
1 & \text { if } \mathrm{f}(\mathrm{x}, \mathrm{y}) \geq 250 \\
0 & \text { Otherwise }<250
\end{array}\right.
$$

Where $f(x, y)$ means grey value which is 1 if greater than or equal 250 and 0 if less than 250 . $B(x, y)$ refers to binary image. Thresholding is a necessary stage for extracting important spots from face. As shown in Fig.3, the binary image has a lot of noise and it is hard to remove it using morphology operation because the goal is to extract the hottest region from face region. Thus, noise removed based on masking each blob with infrared matrix and remove masked blob with lowest temperature as explained in blob refinement section. Blob refinement used for extracting the hottest masked blob was based on it is temperature compare to other blob.

\subsection{Blob Refinement}

The method of blob refinement defined as follows:

Algorithm 1: Blob refinement.

1:load infrared thermal matrix
2:load binary image
3:Obtain Number of blobs
4:For Loop on Number of blobs
5:Masking blob with infrared
thermal matrix
6:Max Temperature for each blob
7:Mean for extracted Max temperature
values
8: If temperature of blob less than
Mean
9: Convert current entire region to zero
10: else
11: check another blob
12 EndIf
13: end for loop


Algorithm (1) for blob refinement start by loading two inputs image: infrared thermal matrix and binary image from previous stage as input. Normally, binary image has two values for each pixel: 1 for blob and zero for background. While infrared matrix has a range of values 20 to 38 Celsius, in binary image, the number of blobs is obtained, and used this number as number loop over all blobs in binary image. Inside loop, masking applied between blob and infrared matrix. After applying masking, Max temperature for each masked blob obtained. The mean of Max temperature values will be used as a threshold for removing masked blob with less temperature and keeping hot masked blobs only from the face region. This is stage is necessary to keep only hot spots from face region. It is assumed as the hottest region that masked blob with high temperature, which is greater than temperature average.

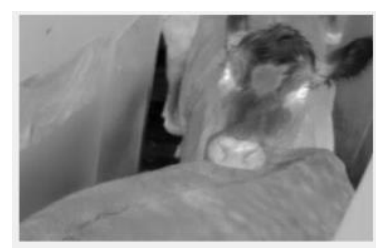

A

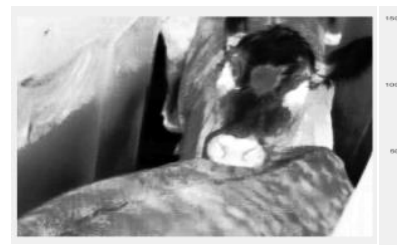

C

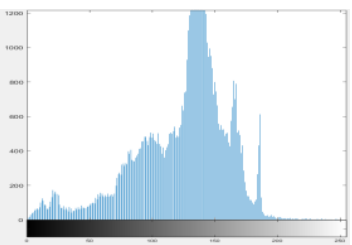

B

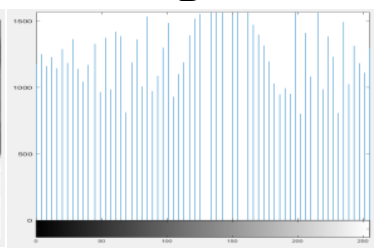

D
Figure 2: Histogram equalization: (A) input image with grayscale, (B) histogram distribution for input image, (C) input image after apply histogram equalization, (D) final image with histogram equalization.

\subsection{Hot Spot Extraction and Temperature Analysis}

The last stage is obtaining a temperature for remaining blobs. Temperature extraction use same method as mentioned in the algorithm (1). Masking between each blob with the infrared thermal matrix. The bounding box will contain remained blob with showing max temperature value as shown in Fig.3 (M,N,P,Q). Remaining blobs represent a hot spot of the face region. Temperature measurement of these blobs can be calculated by using equation (1).

\section{PRELIMINARY RESULTS AND DISCUSSION}

In this paper, face detection is tested only through split infrared thermal database to two group: training and testing. While hot spot extraction was not tested yet as result to require preparing ground truth dataset. Equation (3) refers to evaluation of face detection.

$$
E_{p}=\frac{N_{D}}{N_{I}} * 100
$$

As shown in (3), $E_{p}$ refers to evaluation for the proposed method. $N_{D}$ means number of face detection, which is 120 . While $N_{I}$ represents number of infrared thermal images in the database, which is 150 images. This means face detected correctly with percentage $80 \%$.

As presented in Fig.3 (M,N,P,Q), the temperature for the different spot of face region was in range 32 to 37 Celsius. Eyes region were in range 34 to 37 , while the nose was in range 34 to 36 . We assume hottest spot is region with higher temperature in this case masked blob with higher temperature. These temperature values can be used in future as an indicator of inflammation and disease as mentioned in section .1.

\section{CONCLUSIONS}

In this paper, a method is proposed for temperature extraction from face region in cattle through employing infrared thermal image. The contribution of this paper is the new implementation for face detection in cattle using infrared thermal images. In addition, the new method proposed for hot spot extraction from face region. Unlike evaluation for face detection, we did not evaluate the hot spot extraction due to it is varied from animal to other. As future work, eye and nose detection with correlation to inflammation or disease will be a new path in this research area.

\section{ACKNOWLEDGEMENTS}

This research paper is the result of help and assistance from the team of researchers. Firstly, I would like to thank my supervisor Dr. Adel for his unlimited 
support during the present research. In addition, the Co-supervisor Dr. Luciano helps to provide me with the database. Lastly, I am grateful for my colleague Holly for her answering my enquiries regarding the physiological status of cattle.
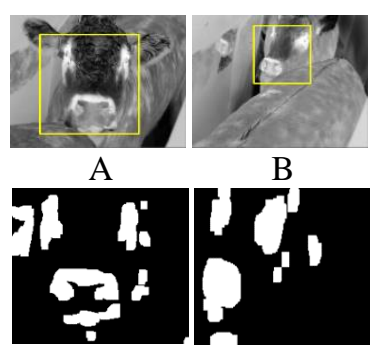

$\mathrm{E}$

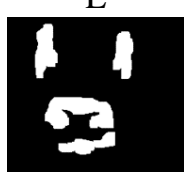

I

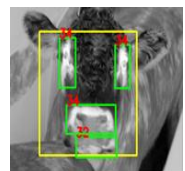

M

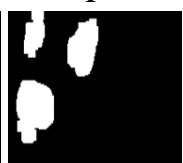

$\mathrm{J}$

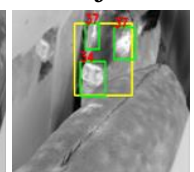

$\mathrm{N}$

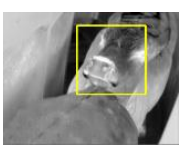

C

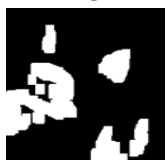

G

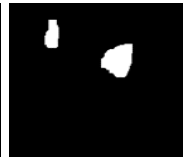

$\mathrm{K}$

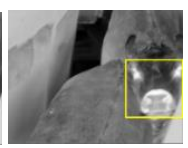

$\mathrm{D}$

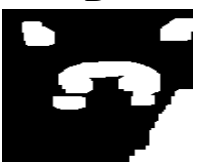

$\mathrm{H}$

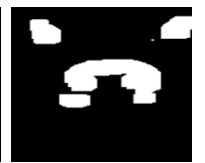

L

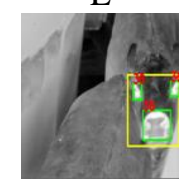

Q
Figure 3: Stages of temperature extraction and analysis for face region.

\section{REFERENCES}

Nääs, I. A., R. G. Garcia and F. R. Caldara (2014). "Infrared thermal image for assessing animal health and welfare." JABB-Online Submission System 2(3): 66-72.

Roberto, J. V. B., B. de Souza, D. A. Furtado, L. J. B. Delfino and B. d. A. Marques (2014). "Thermal gradients and physiological responses of goats in the Brazilian semi-arid using thermography infrared."

Budzan, S. and R. Wyżgolik (2013). "Face and eyes localization algorithm in thermal images for temperature measurement of the inner canthus of the eyes." Infrared Physics \& Technology 60: 225-234.

Cruz-Albarran, I. A., J. P. Benitez-Rangel, R. A. OsornioRios and L. A. Morales-Hernandez (2017). "Human emotions detection based on a smart-thermal system of thermographic images." Infrared Physics \& Technology 81: 250-261.

Dalal, N. and B. Triggs (2005). Histograms of oriented gradients for human detection. Computer Vision and Pattern Recognition, 2005. CVPR 2005. IEEE Computer Society Conference on, IEEE.

Jaddoa, M. A., A. Al-Jumaily, L. Gonzalez and H. Cuthbertson (2017). Automatic eyes localization in thermal images for temperature measurement in cattle. Intelligent Systems and Knowledge Engineering (ISKE), 2017 12th International Conference on, IEEE.
King, D. E. (2009). "Dlib-ml: A machine learning toolkit." Journal of Machine Learning Research 10(Jul): 17551758.

Kopaczka, M., J. Nestler and D. Merhof (2017). Face Detection in Thermal Infrared Images: A Comparison of Algorithm-and Machine-Learning-Based Approaches. International Conference on Advanced Concepts for Intelligent Vision Systems, Springer.

Li, D., C. C. Menassa and V. R. Kamat (2018). "Nonintrusive interpretation of human thermal comfort through analysis of facial infrared thermography." Energy and Buildings 176: 246-261.

Nääs, I. A., R. G. Garcia and F. R. Caldara (2014). "Infrared thermal image for assessing animal health and welfare." JABB-Online Submission System 2(3): 66-72.

Palme, R. and F. S. Schenkel "Relationships between residual feed intake and infrared thermography and glucocorticoid levels in feedlot steers from three different sire breeds."

Pezeshki, A., P. Stordeur, H. Wallemacq, F. Schynts, M. Stevens, P. Boutet, L. J. Peelman, B. De Spiegeleer, L. Duchateau and F. Bureau (2011). "Variation of inflammatory dynamics and mediators in primiparous cows after intramammary challenge with Escherichia coli." Veterinary research 42(1): 15.

Polat, B., A. Colak, M. Cengiz, L. Yanmaz, H. Oral, A. Bastan, S. Kaya and A. Hayirli (2010). "Sensitivity and specificity of infrared thermography in detection of subclinical mastitis in dairy cows." Journal of Dairy Science 93(8): 3525-3532.

Rekant, S. I., M. A. Lyons, J. M. Pacheco, J. Arzt and L. L. Rodriguez (2016). "Veterinary applications of infrared thermography." American journal of veterinary research 77(1): 98-107.

Roberto, J. V. B., B. de Souza, D. A. Furtado, L. J. B. Delfino and B. d. A. Marques (2014). "Thermal gradients and physiological responses of goats in the Brazilian semi-arid using thermography infrared." Journal of Animal Behaviour and Biometeorology 2(1): 11-19.

Schaefer, A., N. Cook, J. Church, J. Basarab, B. Perry, C. Miller and A. Tong (2007). "The use of infrared thermography as an early indicator of bovine respiratory disease complex in calves." Research in Veterinary Science 83(3): 376-384.

Soroko, M. and K. Howell (2018). "Infrared thermography: current applications in equine medicine." Journal of Equine Veterinary Science 60: 90-96. e92.

Stewart, M., J. Webster, G. Verkerk, A. Schaefer, J. Colyn and K. Stafford (2007). "Non-invasive measurement of stress in dairy cows using infrared thermography." Physiology \& Behavior 92(3): 520-525.

Yáñez-Pizaña, A., D. Mota-Rojas, R. Ramírez-Necoechea, M. Castillo-Rivera, P. Roldán-Santiago, P. MoraMedina and M. González-Lozano (2019). "Application of infrared thermography to assess the effect of different types of environmental enrichment on the ocular, auricular pavilion and nose area temperatures of weaned piglets." Computers and Electronics in Agriculture 156: 33-42. 